\title{
THE REPRESENTATION OF A PAIR OF INTEGERS BY A PAIR OF POSITIVE-DEFINITE BINARY QUADRATIC FORMS
}

\author{
KENNETH HARDY, PIERRE KAPLAN AND KENNETH S. WILLIAMS
}

(Communicated by William Adams)

\begin{abstract}
An explicit formula is given for the number of representations of a pair of positive integers by a representative set of inequivalent pairs of integral positive-definite binary quadratic forms with given invariants.
\end{abstract}

\section{Notation}

By a form we mean a binary quadratic form $f=(a, b, c)=a X^{2}+b X Y+c Y^{2}$, which is integral (that is $a, b, c$ are integers), positive definite (that is $a>0$, $\left.b^{2}-4 a c<0\right)$ and primitive (that is $\operatorname{GCD}(a, b, c)=1$ ). The discriminant of $f$, written $\operatorname{disc}(f)$, is the integer $b^{2}-4 a c$.

\section{INTRODUCTION}

Two forms $f$ and $f^{\prime}$ are said to be equivalent (written $f \sim f^{\prime}$ ) if there exists a transformation

$$
\tau:\left(\begin{array}{c}
X \\
Y
\end{array}\right) \rightarrow\left(\begin{array}{ll}
r & s \\
t & u
\end{array}\right)\left(\begin{array}{c}
X \\
Y
\end{array}\right)
$$

where $r, s, t, u$ are integers satisfying $r u-s t=1$, such that

$$
f(r X+s Y, t X+u Y)=f^{\prime}(X, Y) .
$$

The transformation $\tau$ preserves $\operatorname{disc}(f)$. The relation $\sim$ is an equivalence relation on the set of forms with given discriminant $d$. It is well known that the number $h(d)$ of equivalence classes is finite. Let

$$
f_{i}=a_{i} X^{2}+b_{i} X Y+c_{i} Y^{2}, \quad i=1,2, \ldots, h(d),
$$

be a representative set of inequivalent forms of discriminant $d$. The positive

Received by the editors July 28,1988 .

1980 Mathematics Subject Classification (1985 Revision). Primary 10C99.

Research of the first author supported by Natural Sciences and Engineering Research Council of Canada grant A-7823.

Research of the third author supported by Natural Sciences and Engineering Research Council of Canada grant A-7233 and the University of Nancy 1. 
integer $m$ is said to be represented by the form $f_{i}$ if there exist integers $x$ and $y$ such that

$$
m=f_{i}(x, y) \text {. }
$$

The number of pairs $(x, y)$ of integers satisfying $(1.4)$ is denoted by $\psi_{d}^{(i)}(m)$. Clearly $\psi_{d}^{(i)}(m)$ is unchanged if the form $f_{i}$ is replaced by another form equivalent to it. The total number of representations of $m$ by a representative set of inequivalent forms of discriminant $d$ is

$$
\psi_{d}(m)=\sum_{i=1}^{h(d)} \psi_{d}^{(i)}(m) .
$$

In [1] Dirichlet proved that if $G C D(m, 2 d)=1$ then

$$
\psi_{d}(m)=w(d) \sum_{e \mid m}\left(\frac{d}{e}\right),
$$

where $e$ runs through all the positive integers dividing $m,(d / e)$ is the Kronecker symbol and

$$
w(d)= \begin{cases}4, & \text { if } d=-4, \\ 6, & \text { if } d=-3, \\ 2, & \text { if } d \neq-3,-4\end{cases}
$$

In this paper we consider the representability of a pair of positive integers $(m, M)$ by pairs of forms and obtain results analogous to Dirichlet's formula $(1.6)$.

\section{PAIRS OF FORMS}

Two pairs of forms $(f, F)=\left(a x^{2}+b x y+c y^{2}, A x^{2}+B x y+C y^{2}\right)$ and $\left(f^{\prime}, F^{\prime}\right)$ are said to be equivalent, written $(f, F) \sim\left(f^{\prime}, F^{\prime}\right)$, if there exists a transformation $\tau$ of the type given in (1.1) such that

$$
(f(r X+s Y, t X+u Y), F(r X+s Y, t X+u Y))=\left(f^{\prime}(X, Y), F^{\prime}(X, Y)\right) \text {. }
$$

The transformation $\tau$ preserves $d=\operatorname{disc}(f)=b^{2}-4 a c, D=\operatorname{disc}(F)=$ $B^{2}-4 A C$, as well as the codiscriminant $\Delta=\operatorname{codisc}(f, F)=b B-2 a C-2 c A$ of the pair $(f, F)$ [3]. From now on we suppose that $d, D$, and $\Delta$ are given and that there are pairs of forms $(f, F)$ with $\operatorname{disc}(f)=d, \operatorname{disc}(F)=D$, and $\operatorname{codisc}(f, F)=\Delta$. It is easy to prove [2] that

$$
\Delta<0, \quad \Delta^{2}-d D \geq 0 .
$$

If $\Delta^{2}-d D=0$ it is straightforward [2] to show that $d=D=\Delta$ and that any pair $(f, F)$ with these invariants must have $f=F$. Thus in this case equivalence of pairs of forms reduces to the equivalence of forms described in $\S 1$. Thus we may exclude this case and assume from now on that

$$
\Delta^{2}-d D>0 \text {. }
$$


On the set of pairs of forms $(f, F)$ with specified $d, D$, and $\Delta$, the relation $\sim$ is an equivalence relation, and the number $h(d, D, \Delta)$ of equivalence classes is finite [3]. A formula for $h(d, D, \Delta)$ has been given by Hardy and Williams [2] in the case when $d$ and $D$ are fundamental discriminants and $G C D(d D, \Delta)=$ $2^{l}$ for some $l \geq 0$. We let

$$
\begin{aligned}
\left(f_{i}, F_{i}\right)=\left(a_{i} X^{2}+b_{i} X Y+c_{i} Y^{2}, A_{i} X^{2}+B_{i} X Y+C_{i} Y^{2}\right), \\
i=1,2, \ldots, h(d, D, \Delta),
\end{aligned}
$$

be a representative set of inequivalent pairs of forms with given $d, D$, and $\Delta$. We say that the pair $(m, M)$ of positive integers is represented by the pair $\left(f_{i}, F_{i}\right)$ if there exist integers $x, y$ such that

$$
m=f_{i}(x, y), \quad M=F_{i}(x, y) .
$$

The number of pairs of integers $(x, y)$ satisfying $(2.5)$ is denoted by $\Psi_{d, D, \Delta}^{(i)}$ $(m, M)$. Clearly $\Psi_{d, D, \Delta}^{(i)}(m, M)$ is unaltered if the pair $\left(f_{i}, F_{i}\right)$ is replaced by another pair of forms equivalent to $\left(f_{i}, F_{i}\right)$. The total number of representations of $(m, M)$ by a representative set of inequivalent pairs of forms is

$$
\Psi_{d, D, \Delta}(m, M)=\sum_{i=1}^{h(d, D, \Delta)} \Psi_{d, D, \Delta}^{(i)}(m, M) .
$$

We prove the following theorem which gives the value of $\Psi_{d, D, \Delta}(m, M)$ for all positive integers $m, M$ for which

$$
G C D\left(m, 2 d\left(\Delta^{2}-d D\right)\right)=G C D\left(M, 2 D\left(\Delta^{2}-d D\right)\right)=1 .
$$

Theorem. (a) If $d M^{2}-2 \Delta M m+D m^{2}$ is not a square then

$$
\Psi_{d, D, \Delta}(m, M)=0 .
$$

(b) If $d M^{2}-2 \Delta M m+D m^{2}=k^{2}$ for some integer $k$ and

$$
G C D(m, M)=G C D(m, 2 d)=G C D(M, 2 D)=1
$$

then

$$
\Psi_{d, D, \Delta}(m, M)=\left\{\begin{array}{l}
4, \text { if } k \neq 0, \\
2, \text { if } k=0 .
\end{array}\right.
$$

(c) If $d M^{2}-2 \Delta M m+D m^{2}=k^{2}$ for some integer $k$ and

$$
G C D\left(m, 2 d\left(\Delta^{2}-d D\right)\right)=G C D\left(M, 2 D\left(\Delta^{2}-d D\right)\right)=1
$$

\section{then}

$$
\Psi_{d, D, \Delta}(m, M)= \begin{cases}4, & \text { if } k \neq 0 \text { and } G C D(m, M)=l^{2} \text { for some integer } l, \\ 2, & \text { if } k=0 \text { and } G C D(m, M)=l^{2} \text { for some integer } l, \\ 0, & \text { if } G C D(m, M) \neq l^{2} \text { for any integer } l .\end{cases}
$$




\section{Proof of THeOREM (a)}

If $\Psi_{d, D, \Delta}(m, M) \geq 1$ then there are integers $x$ and $y$ and an integer $i(1 \leq$ $i \leq h(d, D, \Delta))$ such that

$$
\left\{\begin{array}{l}
m=a_{i} x^{2}+b_{i} x y+c_{i} y^{2} \\
M=A_{i} x^{2}+B_{i} x y+C_{i} y^{2}
\end{array}\right.
$$

and so

$$
d M^{2}-2 \Delta M m+D m^{2}=k^{2}
$$

where

$$
\pm k=\left(a_{i} B_{i}-b_{i} A_{i}\right) x^{2}+2\left(a_{i} C_{i}-c_{i} A_{i}\right) x y+\left(b_{i} C_{i}-c_{i} B_{i}\right) y^{2} .
$$

Hence if $d M^{2}-2 \Delta M m+D m^{2}$ is not a square, we must have $\Psi_{d, D, \Delta}(m, M)=$ 0 .

\section{Proof OF THEOREM (b)}

Throughout this section we assume that $m, M$ are positive integers satisfying (2.9) and that there exists an integer $k$ such that (3.2) holds. The number of pairs of integers $n(\bmod 2 m)$ and $N(\bmod 2 M)$ such that

$$
n^{2} \equiv d(\bmod 4 m), \quad N^{2} \equiv D(\bmod 4 M),
$$

and for which

$$
\text { there exist representatives satisfying } M n-m N=k \text {, }
$$

is denoted by $A(m, M)$. We begin by determining $A(m, M)$.

Lemma 1. $A(m, M)=1$.

Proof. Clearly, for any solution of (4.1) satisfying (4.2), one has

$$
M n \equiv k(\bmod m), \quad m N \equiv-k(\bmod M) .
$$

Conversely, for any pair of integers $\left(n_{0}, N_{0}\right)$ for which (4.1) and (4.3) hold, we have

$$
\begin{aligned}
& M n_{0}-m N_{0} \equiv k(\bmod m), \\
& M n_{0}-m N_{0} \equiv k(\bmod M), \\
& M n_{0}-m N_{0} \equiv M^{2} n_{0}^{2}+m^{2} N_{0}^{2} \equiv d M^{2}+D m^{2} \equiv k^{2} \equiv k(\bmod 2)(\text { by }(3.2)),
\end{aligned}
$$

and so

$$
M n_{0}-m N_{0} \equiv k(\bmod 2 m M)
$$

Noting that

$$
M\left(n_{0}+2 m r\right)-\left(N_{0}+2 M R\right)=\left(M n_{0}-m N_{0}\right)+2 m M(r-R),
$$

we see that the classes of $n_{0}(\bmod 2 m)$ and $N_{0}(\bmod 2 M)$ contain representatives $n$ and $N$ satisfying $M n-m N=k$, that is (4.2) holds. Thus we have

$$
A(m, M)=B(d, m, M, k) B(D, M, m,-k),
$$


where $B(d, m, M, k)$ is the number of solutions $n(\bmod 2 m)$ of

$$
n^{2} \equiv d(\bmod 4 m), \quad M n \equiv k(\bmod m) .
$$

The congruence $M n \equiv k(\bmod m)$ has a unique solution $n_{0}(\bmod m)$. For this solution the congruence $n_{0}^{2} \equiv d(\bmod m)$ is automatically true in view of (3.2). The solutions $\bmod 2 m$ of $M n \equiv k(\bmod m)$ are given by

$$
n_{0}+\varepsilon m, \varepsilon=0 \text { or } 1 \text {. }
$$

These solutions satisfy $n^{2} \equiv d(\bmod 4)$ for the unique value of $\varepsilon$ such that

$$
\left(n_{0}+\varepsilon\right)^{2} \equiv d(\bmod 4) \text {. }
$$

Thus we have $B(d, m, M, k)=1$ and similarly $B(D, M, m,-k)=1$. Hence (4.4) gives $A(m, M)=1$ as required.

The next lemma gives the automorphs of a pair of forms $(f, F)$.

Lemma 2. The only transformations

$$
\tau:\left(\begin{array}{l}
X \\
Y
\end{array}\right) \rightarrow\left(\begin{array}{ll}
r & s \\
t & u
\end{array}\right)\left(\begin{array}{l}
X \\
Y
\end{array}\right) \quad(r u-s t=1)
$$

mapping the pair of forms $(f, F)$ into itself are given by

$$
\left(\begin{array}{ll}
r & s \\
t & u
\end{array}\right)= \pm\left(\begin{array}{ll}
1 & 0 \\
0 & 1
\end{array}\right)
$$

Proof. If $d \neq-3,-4$ the only automorphs of the form $f=a x^{2}+b x y+c y^{2}$ of discriminant $d$ are

$$
\pm\left(\begin{array}{ll}
1 & 0 \\
0 & 1
\end{array}\right) \text {. }
$$

Thus the assertion of the lemma is clear unless $(d, D)=(-3,-3),(-3,-4)$, $(-4,-3)$ or $(-4,-4)$.

We just treat the case $(d, D)=(-3,-3)$ as the other cases can be treated similarly. As every form of discriminant -3 is equivalent to the form $(1,1,1)$ we may suppose by applying a suitable transformation to $f$ that $f=(1,1,1)$. The only automorphs of $f$ are

$$
\pm\left(\begin{array}{ll}
1 & 0 \\
0 & 1
\end{array}\right), \quad \pm\left(\begin{array}{cc}
0 & 1 \\
-1 & -1
\end{array}\right), \quad \text { and } \quad \pm\left(\begin{array}{cc}
1 & 1 \\
-1 & 0
\end{array}\right) \text {. }
$$

The second of these transforms $F=(A, B, C)$ into $(C,-B+2 C, A-B+C)$ and so can only be an automorph for the pair $(f, F)$ if $A=C, B=-B+2 C$, $C=A-B+C$, that is $A=B=C$, i.e., $F=(1,1,1)$, and thus $d=D=$ $\Delta=-3$ which is impossible as $\Delta^{2}-d D \neq 0$. The third mapping transforms $F=(A, B, C)$ into $(A-B+C, 2 A-B, A)$, and, exactly as above, we see that it cannot be an automorph of the pair $(f, F)$. This completes the proof of Lemma 2.

The next lemma is easily checked. 
Lemma 3. If $d=n^{2}-4 m l, D=N^{2}-4 M L$ then the following is an identity

$$
d M^{2}+D m^{2}-(m N-M n)^{2}=2 m M(n N-2 m L-2 M l) .
$$

We are now ready to prove Theorem (b). If $(x, y)$ is a pair of integers, we set

$$
[x, y]=\{(x, y),(-x,-y)\}
$$

and for $i=1,2, \ldots, h(d, D, \Delta)$ we let

$$
S_{i}=\left\{[x, y] \mid m=a_{i} x^{2}+b_{i} x y+c_{i} y^{2}, M=A_{i} x^{2}+B_{i} x y+C_{i} y^{2}\right\} .
$$

We remark that if $[x, y] \in S_{i}$ then $G C D(x, y)=1$ as $G C D(m, M)=1$. The set of all pairs $([x, y], i)$ with $[x, y] \in S_{i}$ and $i=1,2, \ldots, h(d, D, \Delta)$ is denoted by $S$. Clearly we have

$$
\operatorname{card}(S)=\frac{1}{2} \Psi_{d, D, \Delta}(m, M) .
$$

Recalling that $m$ and $M$ are positive integers satisfying (2.9) and for which $d M^{2}-2 \Delta M m+D m^{2}=k^{2}$ is solvable, we set

$$
\begin{gathered}
C_{m, M}=\left\{(n(\bmod 2 m), N(\bmod 2 M)) \mid n^{2} \equiv d(\bmod 4 m), N^{2} \equiv D(\bmod 4 M),\right. \\
M n-m N= \pm k\} .
\end{gathered}
$$

By Lemma 1 we have

$$
\operatorname{card}\left(C_{m, M}\right)= \begin{cases}2, & \text { if } k \neq 0, \\ 1, & \text { if } k=0 .\end{cases}
$$

Next we define a mapping $T: S \rightarrow C_{m, M}$ as follows: if $[x, y] \in S_{i}$, where $1 \leq i \leq h(d, D, \Delta)$, then

$$
T(([x, y], i))=(n(\bmod 2 m), N(\bmod 2 M)),
$$

where

$$
n=2 a_{i} x \mu+b_{i}(x \lambda+y \mu)+2 c_{i} y \lambda, \quad N=2 A_{i} x \mu+B_{i}(x \lambda+y \mu)+2 C_{i} y \lambda,
$$

and $\lambda, \mu$ are integers such that

$$
\lambda x-\mu y=1 .
$$

We must show that $T$ is well defined and that $\operatorname{range}(T) \subseteq C_{m, M}$. To see that $T$ is well defined we have only to note that if $(\lambda, \mu)$ is replaced by another solution $(\lambda+t y, \mu+t x)$ of $(4.10)$ then $n$ and $N$ are unchanged $(\bmod 2)$, and if $(x, y)$ is replaced by $(-x,-y)$ then $(\lambda, \mu)$ can be replaced by $(-\lambda,-\mu)$ and $n$ and $N$ remain the same.

Next we show that $T$ maps into $C_{m, M}$. By the transformation

$$
\left(\begin{array}{ll}
x & \mu \\
y & \lambda
\end{array}\right)
$$


the pair of forms $\left(\left(a_{i}, b_{i}, c_{i}\right),\left(A_{i}, B_{i}, C_{i}\right)\right)$ becomes the pair $((m, n, l)$, $(M, N, L))$, where

$$
l=\frac{n^{2}-d}{4 m}, \quad L=\frac{N^{2}-D}{4 M},
$$

and so $n^{2} \equiv d(\bmod 4 m), N^{2} \equiv D(\bmod 4 M)$. As $\Delta=n N-2 m L-2 M l$, by (3.2) and Lemma 3, we have $M n-m N= \pm k$.

Now we prove that $T$ maps onto $C_{m, M}$. Let $((n(\bmod 2 m), N(\bmod 2 M)) \in$ $C_{m, M}$ so that $n^{2} \equiv d(\bmod 4 m), N^{2} \equiv D(\bmod 4 M), M n-m N= \pm k$. We define integers $l, L$ as in (4.11). The forms $(m, n, l)$ and $(M, N, L)$ have discriminants $d$ and $D$, respectively, and, by Lemma 3 and (3.2), their codiscriminant is $\Delta$. Hence, for a unique integer $i(1 \leq i \leq h(d, D, \Delta))$, we have

$$
((m, n, l),(M, N, L)) \sim\left(\left(a_{i}, b_{i}, c_{i}\right),\left(A_{i}, B_{i}, C_{i}\right)\right) .
$$

If

$$
\left(\begin{array}{ll}
x & \mu \\
y & \lambda
\end{array}\right)
$$

where $\lambda x-\mu y=1$, is a transformation mapping $\left(\left(a_{i}, b_{i}, c_{i}\right),\left(A_{i}, B_{i}, C_{i}\right)\right)$ into $((m, n, l),(M, N, L))$ then $[x, y] \in S_{i}$, and $T(([x, y], i))=(n(\bmod 2 m)$, $N(\bmod 2 M))$. This proves that $\operatorname{range}(T)=C_{m, M}$.

Finally we show that $T$ is one-to-one. Suppose that

$$
T([x, y], i)=T\left(\left[x^{\prime}, y^{\prime}\right], i^{\prime}\right) \text {. }
$$

Then there exist integers $n, N, n^{\prime}, N^{\prime}, t, T$ and two transformations

$$
\tau=\left(\begin{array}{cc}
x & \mu \\
y & \lambda
\end{array}\right)(x \lambda-\mu y=1), \quad \tau^{\prime}=\left(\begin{array}{cc}
x^{\prime} & \mu^{\prime} \\
y^{\prime} & \lambda^{\prime}
\end{array}\right)\left(x^{\prime} \lambda^{\prime}-y^{\prime} \mu^{\prime}=1\right)
$$

such that

$$
\begin{gathered}
n=n^{\prime}+2 t m, \quad N=N^{\prime}+2 T M, \\
\left(\left(a_{i}, b_{i}, c_{i}\right),\left(A_{i}, B_{i}, C_{i}\right)\right) \stackrel{\tau}{\rightarrow}((m, n, l),(M, N, L)), \\
\left(\left(a_{i^{\prime}}, b_{i^{\prime}}, c_{i^{\prime}}\right),\left(A_{i^{\prime}}, B_{i^{\prime}}, C_{i^{\prime}}\right)\right) \stackrel{\tau^{\prime}}{\rightarrow}\left(\left(m, n^{\prime}, l^{\prime}\right),\left(M, N^{\prime}, L^{\prime}\right)\right), \\
M n-m N= \pm k, \quad M n^{\prime}-m N^{\prime}= \pm k,
\end{gathered}
$$

where $l, L$ are defined as in (4.11), and $l^{\prime}, L^{\prime}$ are defined similarly. Clearly $M n-m N= \pm\left(M n^{\prime}-m N^{\prime}\right)$ and we show that

$$
M n-m N=M n^{\prime}-m N^{\prime} \text {. }
$$

For otherwise $M n-m N=-\left(M n^{\prime}-m N^{\prime}\right)$ and appealing to (4.12) we obtain $m M(T-t)=M n-m N$. As $G C D(m, M)=1$ we see that $m \mid n$ and $M \mid N$, and so by (4.15) we have $m M \mid k$. Hence from (3.2) we have $m \mid d$ and $M \mid d$, contradicting $G C D(m, 2 d)=G C D(M, 2 D)=1$. This proves (4.16). From (4.12) and (4.16) we deduce that $t=T$ and so

$$
\theta=\left(\begin{array}{cc}
1 & 2 t \\
0 & 1
\end{array}\right)
$$


maps $((m, n, l),(M, N, L)) \rightarrow\left(\left(m, n^{\prime}, l^{\prime}\right),\left(M, N^{\prime}, L^{\prime}\right)\right)$, proving that $i=$ $i^{\prime}$, and that $\tau^{\prime^{-1}} \theta \tau$ is an automorphism of the pair $\left(\left(a_{i}, b_{i}, c_{i}\right),\left(A_{i}, B_{i}, C_{i}\right)\right)$. Hence by Lemma 2 we have

$$
\left(\begin{array}{cc}
1 & 2 t \\
0 & 1
\end{array}\right)\left(\begin{array}{ll}
x & \mu \\
y & \lambda
\end{array}\right)= \pm\left(\begin{array}{cc}
x^{\prime} & \mu^{\prime} \\
y^{\prime} & \lambda^{\prime}
\end{array}\right)\left(\begin{array}{ll}
1 & 0 \\
1 & 1
\end{array}\right)
$$

implying $\left[x^{\prime}, y^{\prime}\right]=[x, y]$. This completes the proof that $T$ is one-to-one.

Thus $T$ is a bijection from $S$ to $C_{m, M}$ and so by (4.6) and (4.7) we have

$$
\frac{1}{2} \Psi_{d, D, \Delta}(m, M)=\operatorname{card}(S)=\operatorname{card}\left(C_{m, M}\right)= \begin{cases}2, & \text { if } k \neq 0, \\ 1, & \text { if } k=0,\end{cases}
$$

completing the proof of Theorem (b).

\section{Proof of THEOREM (c)}

Throughout this section we assume that $m, M$ are positive integers satisfying (3.2) and (2.11).

First we show that if $G C D(m, M) \neq l^{2}$ for any integer $l$ then $\Psi_{d, D, \Delta}(m, M)$ $=0$. For suppose $\Psi_{d, D, \Delta}(m, M) \geq 1$. Then there exists $i(1 \leq i \leq h(d, D, \Delta))$ and integers $x, y$ such that

$$
\begin{aligned}
& m=a_{i} x^{2}+b_{i} x y+c_{i} y^{2}, \\
& M=A_{i} x^{2}+B_{i} x y+C_{i} y^{2} .
\end{aligned}
$$

Also from (3.3) we have

$$
\pm k=\left(a_{i} B_{i}-b_{i} A_{i}\right) x^{2}+2\left(a_{i} C_{i}-c_{i} A_{i}\right) x y+\left(b_{i} C_{i}-c_{i} B_{i}\right) y^{2} .
$$

Solving (5.1) and (5.2) for $x^{2}, x y$ and $y^{2}$, we obtain

$$
\begin{aligned}
& \left(\Delta^{2}-d D\right) x^{2}=2\left(c_{i} D-C_{i} \Delta\right) m+2\left(C_{i} d-c_{i} \Delta\right) M \mp 2 k\left(b_{i} C_{i}-c_{i} B_{i}\right), \\
& \left(\Delta^{2}-d D\right) x y=\left(B_{i} \Delta-b_{i} D\right) m+\left(b_{i} \Delta-B_{i} d\right) M \pm 2 k\left(a_{i} C_{i}-c_{i} A_{i}\right), \\
& \left(\Delta^{2}-d D\right) y^{2}=2\left(a_{i} D-A_{i} \Delta\right) m+2\left(A_{i} d-a_{i} \Delta\right) M \mp 2 k\left(a_{i} B_{i}-b_{i} A_{i}\right) .
\end{aligned}
$$

As $G C D(m, M)$ is not a square, there exists a prime $p$ and a non-negative integer $r$ such that $p^{2 r+1} \| G C D(m, M)$. As $m$ and $M$ are odd we have $p \neq 2$. Further from (3.2) we see that $p^{2 r+1} \mid k$ and so from (5.3) we have

$$
p^{2 r+1}\left|\left(\Delta^{2}-d D\right) x^{2}, \quad p^{2 r+1}\right|\left(\Delta^{2}-d D\right) y^{2} .
$$

By (2.11) we have $p \nmid \Delta^{2}-d D$ and so $p^{r+1} \mid x$ and $p^{r+1} \mid y$. Thus from (5.4) we have $p^{2 r+2} \mid m$ and $p^{2 r+2} \mid M$ contradicting $p^{2 r+1} \| G C D(m, M)$.

Finally, if $G C D(m, M)=l^{2}$, for some integer $l$, then it is easy to check using (5.1), (5.2), and (5.3) that the mapping $(x, y) \rightarrow(x / l, y / l)$ is a bijection from the set of representations of $(m, M)$ by a set of inequivalent pairs of 
forms with invariants $d, D, \Delta$ and the set of representations of $\left(m / l^{2}, M / l^{2}\right)$ by the same set of pairs of forms. Thus we have, by Theorem (b),

$$
\begin{aligned}
\Psi_{d, D, \Delta}(m, l) & =\Psi_{d, D, \Delta}\left(m / l^{2}, M / l^{2}\right)= \begin{cases}4, & \text { if } k / l^{2} \neq 0, \\
2, & \text { if } k / l^{2}=0,\end{cases} \\
& = \begin{cases}4, & \text { if } k \neq 0, \\
2, & \text { if } k=0,\end{cases}
\end{aligned}
$$

as required. This completes the proof of Theorem (c).

\section{AN EXAMPLE}

We take $d=-11, D=-11, \Delta=-19$ so that $\Delta^{2}-d D=240$. Every pair of forms with these invariants is equivalent to exactly one of the pairs

$$
\begin{gathered}
((1,1,3),(3,1,1)), \\
((1,1,3),(3,5,3)), \\
((1,1,3),(1,-3,5)), \\
((1,1,3),(1,5,9)),
\end{gathered}
$$

so $h(-11,-11,-19)=4$.

If we take $m=97$ and $M=31$ (so that $G C D(m, M)=G C D(m, 2 d)=$ $G C D(M, 2 D)=1)$ we have $d M^{2}-2 \Delta M m+D m^{2}=196$, so $k= \pm 14$. Thus by Theorem (b) we must have $\Psi_{-11,-11,-19}(97,31)=4$. Indeed

$$
\begin{array}{lll}
97=x^{2}+x y+3 y^{2}, & 31=x^{2}-3 x y+5 y^{2}, & \text { with }(x, y)= \pm(7,3), \\
97=x^{2}+x y+3 y^{2}, & 31=x^{2}+5 x y+9 y^{2}, & \text { with }(x, y)= \pm(10,-3) .
\end{array}
$$

Finally, we remark that the choice $m=M=3$ shows that the condition $G C D\left(m, \Delta^{2}-d D\right)=G C D\left(M, \Delta^{2}-d D\right)=1$ is necessary in Theorem (c) as

$$
3=x^{2}+x y+3 y^{2}=3 x^{2}+x y+y^{2}
$$

is solvable with $(x, y)= \pm(1,-1)$.

\section{REFERENCES}

1. P. G. L. Dirichlet, Vorlesungen über Zahlentheorie, reprinted 4th edition, Chelsea Publishing Company, New York, 1968, p. 229.

2. K. Hardy and K. S. Williams, The class number of pairs of positive-definite binary quadratic forms, Acta Arithmetica (to appear).

3. C. Hooley, On the diophantine equation $a x^{2}+b y^{2}+c z^{2}+2 f y z+2 g z x+2 h x y=0$, Arch. Math. 19 (1968), 472-478.

Current address (K. Hardy and K. S. Williams): Department of Mathematics and Statistics, Carleton University, Ottawa, Ontario, Canada K1S 5B6

Current address (P. Kaplan) : U.E.R. Des Sciences Mathématiques, Université de Nancy 1 B.P. 239,54506 Vandoeuvre les Nancy, France 